\title{
Changes of cutaneous sensory thresholds induced by non-painful transcutaneous electrical nerve stimulation in normal subjects and in subjects with chronic pain
}

\author{
MASSIMOZOPPI, * FABIO FRANCINI, $\dagger$ MARCO MARESCA, * \\ A N D P A O O PROCACCI* \\ From the Cattedra di Terapia Medica Sistematica, Centro di Algologia, ${ }^{*}$ and the \\ Cattedra di Fisiologia Generale, $\uparrow$ University of Florence, Italy
}

S U M MARY Transcutaneous electrical nerve stimulation (TENS) of the nervi cutaneus surae medialis was applied to 59 healthy subjects and 30 patients suffering from chronic myofascial pain in one lower limb, with an intensity of current that induced a well tolerated tingling sensation. Each period of stimulation lasted 24 minutes. The thresholds of the tactile, tingling and painful sensations were tested at fixed intervals before, during and after stimulation. Trains of constant current square waves in the distribution area of the stimulated nerve (local thresholds) and in other areas (general thresholds) were used. In all subjects repeated changes of the current were necessary in order to maintain constant tingling during the first period of TENS (changing phase); after that few if any changes of the current were necessary (steady phase). There were changes in thresholds within the territory of the electrically stimulated nerve, and marked changes elsewhere and generally in the body. In healthy subjects local thresholds increased during both phases of TENS; general thresholds decreased during the changing phase and increased during the steady phase. After TENS, thresholds showed the same trend as during the steady phase. Trends of the sensory thresholds during and after TENS differed in different subjects according to their thresholds before TENS. Thresholds did not return to normal for more than 20 minutes after TENS. In the group of 30 patients there was a significant difference between thresholds on the two sides of the body. The difference between the two sides was reduced by TENS. Pain relief induced by TENS may be related to this fact.

Prolonged transcutaneous electrical nerve stimulation (TENS) is widely used to induce hypoalgesia and pain relief. ${ }^{1-5}$ On previous occasions we have observed the best parameters of stimulation for inducing hypoalgesia to be square waves of $1 \mathrm{~ms}$ at a frequency of $50 \mathrm{~Hz}$ with an intensity that induces a well tolerated tingling sensation. ${ }^{6}$

Sensory changes induced by TENS have been studied by various methods. ${ }^{3-10}$ Our report analyses the effects of TENS on sensory thresholds in normal subjects and in patients with chronic myofascial pain. We measured sensory thresholds in the distribution area of the stimulated nerve

Address for reprint requests: Dr $\mathbf{P}$ Procacci, Cattedra di Terapia Medica Sistematica, Istituto di Clinica Medica I. Universiià di Firenze, Viale Morgagni 85, I-50134 Firenze, Italy

Accepted 22 May 1981. and in all four limbs. In this way we investigated whether the possible effects of TENS on sensory thresholds are general, segmental or unilateral.

Previous investigations of pain threshold determined with radiant heat ${ }^{11} 12$ and of tactile, tingling and pain thresholds determined with short trains of electrical stimuli ${ }^{13} 14$ have been carried out on healthy subjects and on subjects with pain in one limb. No differences were found between the right and the left limbs in normal subjects. ${ }^{113}$ We observed that in most subjects with pain the sensory thresholds on the painful limb side were higher or lower than on the opposite side..$^{12} 14$

We determined sensory thresholds by electrical stimuli, for two different purposes: for inducing a long lasting tingling sensation resulting in hypoalgesia and pain relief; and for testing sensory thresholds in different areas of the body using 
short electrical pulses. These methods have the following advantages: they can be easily quantified and applied to any part of the body surface; the stimuli normally used do not cause damage and can therefore be repeated; the sensory thresholds induced by electrical stimulation are constant and reproducible. ${ }^{15}$ Thermal stimuli (radiant heat) cannot, however, be repeated in the same skin area at short intervals as this causes cutaneous hyperalgesia and tissue damage.

\section{Materials and methods}

We studied 59 healthy subjects, 37 males and 22 females, between 22 and 48 years of age, and 30 patients, 18 males and 12 females, between 26 and 55 years of age. All patients suffered from nonarticular rheumatism ("fibrositis"), ${ }^{16}$ with myofascial pain in one lower limb, with an evident trigger point at the musculo-tendinous junction of $m$ triceps surae with the Achilles tendon. None of the patients suffered from systemic disease, occlusive arterial disease or neurological disturbance. The experiments were carried out with the subjects in a comfortable position. Room temperature was $20-24^{\circ} \mathrm{C}$. Changes in light intensity and noise were avoided. After adequate instruction and training of all subjects, we measured their sensory thresholds with constant current stimuli applied to the skin by means of $\mathrm{Ag} / \mathrm{AgCl} / \mathrm{KCl}$ surface electrodes of $8 \mathrm{~mm}$ diameter. The applied stimuli were $20 \mathrm{~ms}$ trains of $1 \mathrm{~ms}$ square waves at a frequency of $250 \mathrm{~Hz}$, repeated every second. Three sensations were induced on progressively increasing the intensity of the current: touch, tingling, pain. ${ }^{13}$ Of these sensations we evaluated the corresponding thresholds with the method of limits, that is the intensity of the current was increased until the subject perceived the sensation, and subsequently decreased until the sensation disappeared. The threshold was the mean of the value just above, plus the value just below, the sensation.

Transcutaneous electrical nerve stimulation (TENS) was applied with $\mathrm{Ag} / \mathrm{AgCl} / \mathrm{KCl}$ surface electrodes $3 \times 7 \mathrm{~cm}$. The cathode was applied over the $\mathrm{n}$ cutaneus surae medialis at the level of the musculo-tendineous junction of the $m$ triceps surae with the Achilles tendon, corresponding to the trigger point area in the patients, and the anode on the anterior surface of the leg. In these patients TENS was applied always to the painful limb. The pulses were constant current rectangular waves of $1 \mathrm{~ms}$ at a frequency of $50 \mathrm{~Hz}$. Using these parameters, stimulation induced a welltolerated tingling sensation and a good level of analgesia. ${ }^{6}$ The subjects felt tingling throughout the whole posterior surface of the leg distal to the electrode. During TENS, the intensity of the current was changed in order to maintain the tingling sensation constant; the application lasted 24 minutes.

Sensory thresholds were measured: (1) in the distribution area of the stimulated nerve, that is on the leg $10 \mathrm{~cm}$ distally to the stimulating cathode: local thresholds; (2) outside the distribution area, that is on the anterior surface of both legs and on the volar surface of both forearms: general thresholds. The sensory threshold tests were repeated every $4 \mathrm{~min}$, three times immediately before TENS, during TENS and for $20 \mathrm{~min}$ after TENS. At this point we terminated the test to avoid mental fatigue in the subjects.

The subjects were also able to feel the tingling sensation in the threshold test during TENS because the tingling induced by TENS at a frequency of $50 \mathrm{~Hz}$ is gross, while that induced for threshold determination at a frequency of $250 \mathrm{~Hz}$ is fine. Patients took no analgesics during the week preceding the tests.

\section{Results}

\section{Normal subjects}

Subjects were divided into three groups on the basis of the mean value of the pain threshold in the lower limbs before TENS: (1) subjects with pain thresholds below $2.5 \mathrm{~mA}$ (low thresholds) (13 subjects); (2) subjects with pain thresholds above $2.5 \mathrm{~mA}$ and below $3.5 \mathrm{~mA}$ (medium thresholds) (21 subjects); (3) subjects with pain thresholds above $3.5 \mathrm{~mA}$ (high thresholds) (25 subjects). There were no differences between the left and the right side. Sensory thresholds (except the tactile threshold in the group with low thresholds) were higher in the legs than in the forearms. This finding is similar to our previous experimental observations on cutaneous pain threshold, using thermal and electrical stimuli. ${ }^{13} 17$

During the first 10 minutes of stimulation, repeated increases in the TENS current were necessary to maintain a constant tingling sensation. These increases were large in the low threshold group, small in the high threshold group and intermediate in the medium threshold group. We call this the "changing" or "unsteady" phase of TENS. After this phase, few if any changes of the current were necessary to maintain constant tingling. We call this the "steady phase" of TENS (table 1).

Sensory thresholds in the distribution area of the stimulated nerve (local thresholds) increased during TENS. The increase induced by TENS was small during the changing phase and great during the steady phase. This increase was highest in the low threshold group and lowest in the high threshold group. After TENS the local sensory thresholds decreased but remained significantly higher than before TENS for $10 \mathrm{~min}$ (fig 1; table 1).

Sensory thresholds outside the distribution area of the stimulated nerve (general thresholds) decreased during the changing phase of TENS. The 
Table 1 Changes of sensory thresholds related to TENS in healthy subjects

\begin{tabular}{|c|c|c|c|c|c|c|c|c|c|}
\hline & & & & \multicolumn{2}{|c|}{ Low thresholds (13) } & \multicolumn{2}{|c|}{ Medium thresholds (2I) } & \multicolumn{2}{|c|}{ High thresholds (25) } \\
\hline & & & & Ipsi & Contra & Ipsi & Contra & Ipsi & Contra \\
\hline \multirow[t]{2}{*}{$\begin{array}{l}\text { A: } \\
\text { Changing } \\
\text { phase of TENS }\end{array}$} & $\begin{array}{l}\text { General } \\
\text { thresholds }\end{array}$ & $\begin{array}{l}\text { Fore-arm } \\
\text { Leg }\end{array}$ & 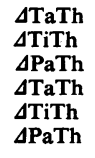 & $\begin{array}{r}-9 \pm 2 \\
-9 \pm 2 \\
-10 \pm 2 \\
-8 \pm 3 \\
-10 \pm 3 \\
-12 \pm 4\end{array}$ & $\begin{array}{r}-9 \pm 3 \\
-8 \pm 2 \\
-9 \pm 2 \\
-8 \pm 4 \\
-9 \pm 3 \\
-13 \pm 4\end{array}$ & $\begin{array}{l}-38 \pm 3 \\
-36 \pm 3 \\
-29 \pm 3 \\
-34 \pm 4 \\
-37 \pm 2 \\
-30 \pm 3\end{array}$ & $\begin{array}{l}-39 \pm 3 \\
-37 \pm 3 \\
-29 \pm 3 \\
-36 \pm 3 \\
-32 \pm 2 \\
-31 \pm 2\end{array}$ & $\begin{array}{l}-62 \pm 2 \\
-57 \pm 2 \\
-52 \pm 2 \\
-65 \pm 2 \\
-60 \pm 3 \\
-55 \pm 3\end{array}$ & $\begin{array}{l}-64 \pm 3 \\
-56 \pm 2 \\
-53 \pm 3 \\
-63 \pm 2 \\
-57 \pm 2 \\
-56 \pm 3\end{array}$ \\
\hline & $\begin{array}{l}\text { Local } \\
\text { thresholds }\end{array}$ & & $\begin{array}{l}\Delta \mathrm{TaTh} \\
\Delta \mathrm{TiTh} \\
\Delta \mathrm{PaTh} \\
\Delta \mathrm{I}\end{array}$ & $\begin{array}{c}+18 \pm 3 \\
+16 \pm 3 \\
+10 \pm 4 \\
+200 \pm 19\end{array}$ & & $\begin{array}{c}+15 \pm 3 \\
+11 \pm 2 \\
+6 \pm 3 \\
+80 \pm 16\end{array}$ & & $\begin{array}{c}+8 \pm 2 \\
+6 \pm 2 \\
+3 \cdot 5 \pm 1 * \\
+64 \pm 17\end{array}$ & \\
\hline \multirow[t]{2}{*}{$\begin{array}{l}\text { B: } \\
\text { Steady phase } \\
\text { of TENS }\end{array}$} & $\begin{array}{l}\text { General } \\
\text { thresholds }\end{array}$ & $\begin{array}{l}\text { Forearm } \\
\text { Leg }\end{array}$ & 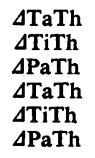 & $\begin{array}{l}+28 \pm 3 \\
+24 \pm 3 \\
+25 \pm 2 \\
+30 \pm 3 \\
+25 \pm 2 \\
+27 \pm 2\end{array}$ & $\begin{array}{l}+31 \pm 2 \\
+27 \pm 3 \\
+28 \pm 1 \\
+33 \pm 3 \\
+30 \pm 3 \\
+31 \pm 2\end{array}$ & $\begin{array}{l}-28 \pm 1 \\
-27 \pm 3 \\
-18 \pm 3 \\
-30 \pm 3 \\
-28 \pm 1 \\
-18 \pm 2\end{array}$ & $\begin{array}{l}-26 \pm 1 \\
-26 \pm 2 \\
-16 \pm 2 \\
-28 \pm 2 \\
-25 \pm 1 \\
-16 \pm 2\end{array}$ & $\begin{array}{l}-58 \pm 2 \\
-44 \pm 3 \\
-43 \pm 2 \\
-58 \pm 4 \\
-45 \pm 2 \\
-42 \pm 3\end{array}$ & $\begin{array}{l}-51 \pm 2 \\
-43 \pm 2 \\
-40 \pm 3 \\
-50 \pm 4 \\
-42 \pm 3 \\
-38 \pm 2\end{array}$ \\
\hline & $\begin{array}{l}\text { Local } \\
\text { thresholds }\end{array}$ & & $\begin{array}{l}\Delta \mathrm{TaTh} \\
\Delta \mathrm{TiTh} \\
\Delta \mathrm{PaTh} \\
\Delta \mathrm{I}\end{array}$ & $\begin{array}{l}+290 \pm 15 \\
+210 \pm 10 \\
+120 \pm 9 \\
+250 \pm 20\end{array}$ & & $\begin{array}{l}+130 \pm 10 \\
+100 \pm 8 \\
+50 \pm 10 \\
+100 \pm 19\end{array}$ & & $\begin{array}{l}+82 \pm 12 \\
+58 \pm 10 \\
+28 \pm 5 \\
+80 \pm 15\end{array}$ & \\
\hline \multirow[t]{2}{*}{$\begin{array}{l}\text { C: } \\
\text { After T ENS }\end{array}$} & $\begin{array}{l}\text { General } \\
\text { thresholds }\end{array}$ & Forearm & $\begin{array}{l}\Delta \text { TaTh } \\
\Delta \text { TiTh } \\
\Delta \text { PaTh } \\
\Delta \text { TaTh } \\
\Delta \text { TiTh } \\
\Delta \text { PaTh }\end{array}$ & $\begin{array}{l}+32 \pm 4 \\
+38 \pm 3 \\
+38 \pm 4 \\
+32 \pm 2 \\
+38 \pm 2 \\
+39 \pm 7\end{array}$ & $\begin{array}{l}+32 \pm 3 \\
+35 \pm 4 \\
+35 \pm 5 \\
+35 \pm 4 \\
+30 \pm 1 \\
+31 \pm 2\end{array}$ & $\begin{array}{l}-29 \pm 2 \\
-26 \pm 1 \\
-16 \pm 1 \\
-28 \pm 3 \\
-25 \pm 2 \\
-16 \pm 2\end{array}$ & $\begin{array}{l}-25 \pm 3 \\
-25 \pm 2 \\
-14 \pm 1 \\
-26 \pm 3 \\
-22 \pm 2 \\
-14 \pm 2\end{array}$ & $\begin{array}{l}-60 \pm 3 \\
-49 \pm 4 \\
-47 \pm 3 \\
-62 \pm 4 \\
-50 \pm 3 \\
-45 \pm 4\end{array}$ & $\begin{array}{l}-56 \pm 3 \\
-45 \pm 4 \\
-44 \pm 3 \\
-59 \pm 4 \\
-45 \pm 3 \\
-40 \pm 4\end{array}$ \\
\hline & $\begin{array}{l}\text { Local } \\
\text { thresholds }\end{array}$ & & $\begin{array}{l}\Delta \mathrm{TaTh} \\
\Delta \mathrm{TiTh} \\
\Delta \mathrm{PaTh}\end{array}$ & $\begin{array}{l}+82 \pm 7 \\
+71 \pm 5 \\
+55 \pm 5\end{array}$ & & $\begin{array}{l}+50 \pm 5 \\
+30 \pm 4 \\
+16 \pm 3\end{array}$ & & $\begin{array}{l}+20 \pm 3 \\
+15 \pm 1 \\
+12 \pm 2\end{array}$ & \\
\hline
\end{tabular}

Mean \pm SE $\quad \Delta$ Ths $=\left(\right.$ Ths - Ths $\left._{0} / T^{T} s_{0}\right) \times 100 ; \quad \Delta I=\left(I-I_{0} / I_{0}\right) \times 100$.

A: General and local sensory thresholds and current intensity of TENS during the changing phase. The sample shown was the change at the 4th min of TENS.

B: General and local sensory thresholds and current intensity of TENS during the steady phase. Figure given is the average of the values during the last $10 \mathrm{~min}$ of TENS.

C: General and local sensory thresholds after TENS. Figure given is the average of the values during the first $10 \mathrm{~min}$ after TENS. General thresholds determined outside the distribution area of the stimulated nerve. Local thresholds: thresholds determined in the distribution number area of the stimulated nerve. In brackets: number of subjects. Ipsi $=$ limbs ipsilateral to TENS ; Contra $=$ limbs contralateral to TENS; TaTh $=$ tactile threshold; TiTh = tingling threshold; PaTh = pain threshold. The thresholds are expressed as changes ( $\Delta T h s)$ from the values before TENS $\left(T_{h s_{0}}\right) . \Delta I=$ changes of the intensity of TENS from $I_{0} . I_{0}$ is the value of the current at which the subjects begin feeling a sharp tingling sensation. Ths $=$ threshold. ${ }^{*}=$ not significant.

Fig 1 Changes of sensory thresholds in the distribution area of the $n$ cutaneus surae medialis (local threholds) before, during and after TENS in a healthy subject with medium sensory thresholds before TENS. TaTh=tactile threshold; $T i T h=$ tingling threshold; PaTh=pain threshold. Ordinate: threshold changes. Tho=average of the values of sensory thresholds before TENS. Abscissa: time. The heavy line is the period of TENS.

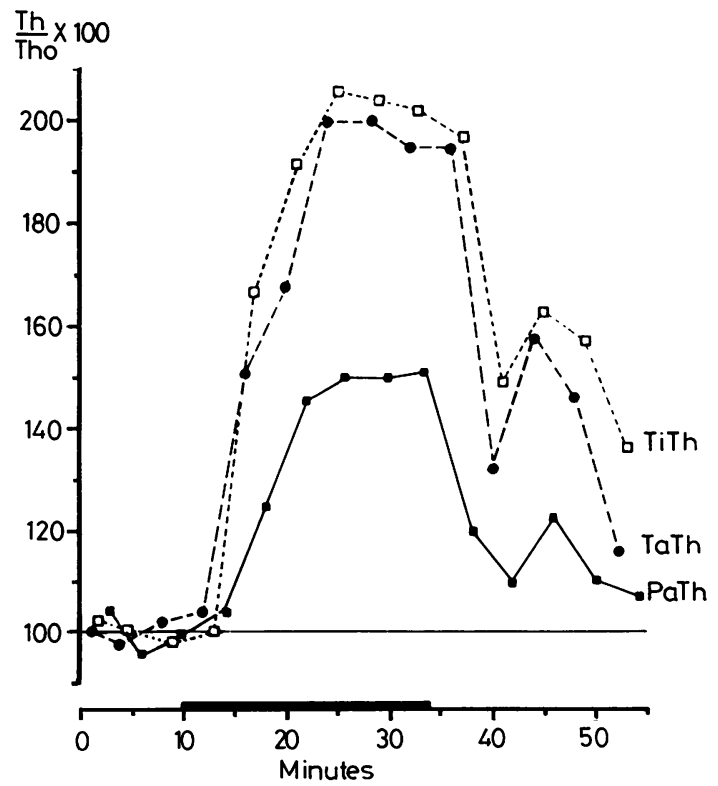


decrease was slight in subjects with low sensory thresholds, intermediate in the medium group and great in subjects with high sensory thresholds. During the steady phase of TENS, general thresholds increased until a value was reached that was higher than before TENS in the group with low thresholds, and lower than before TENS in the groups with medium and high thresholds. After TENS the general thresholds did not change, showing the same trend as during the steady phase for at least 20 min (figs 2,3; table 1).

\section{Patients}

We observed a difference between the sensory thresholds of the patients' painful side and the opposite side $(p<0.001)$ before TENS. We divided the patients into two groups: those with lower sensory thresholds on the affected side (IPSI $<$ CONTRA, 18 subjects) and those with higher sensory thresholds on the affected side (IPSI> CONTRA, 12 subjects). The mean differences $( \pm \mathrm{SE})$ between the thresholds of the two sides, expressed as a percentage of the thresholds of the
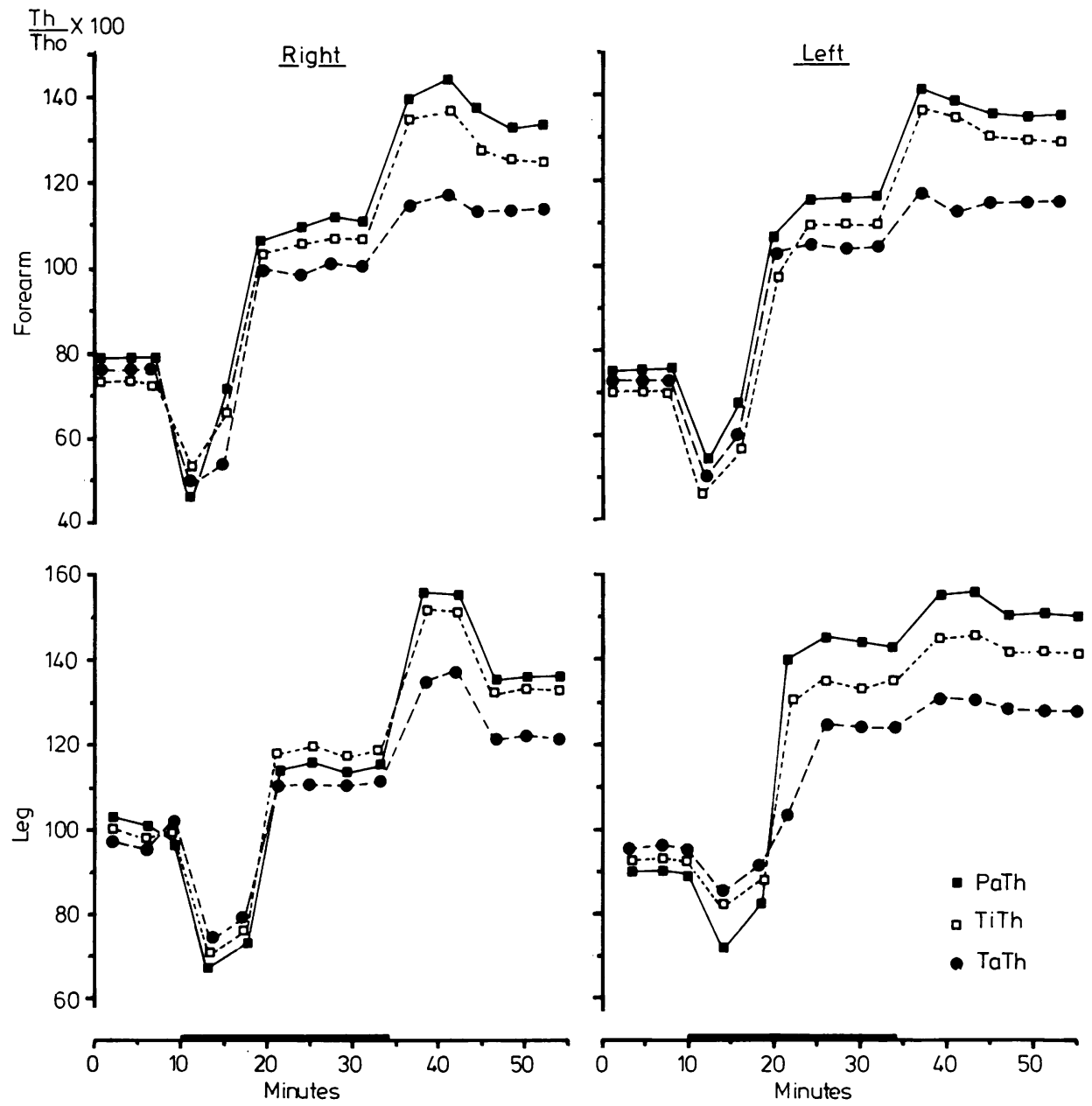

Fig 2 Changes of sensory thresholds outside the distribution area of the stimulated nerve in the four limbs (general thresholds) before, during and after TENS to the right lower limb of a healthy subject with low thresholds before TENS. TaTh, TiTh, PaTh: as in fig 1. Ordinate: changes of sensory thresholds. Tho is the mean value in the right lower limb before TENS. Abscissa: time. The heavy line is the period of TENS. 


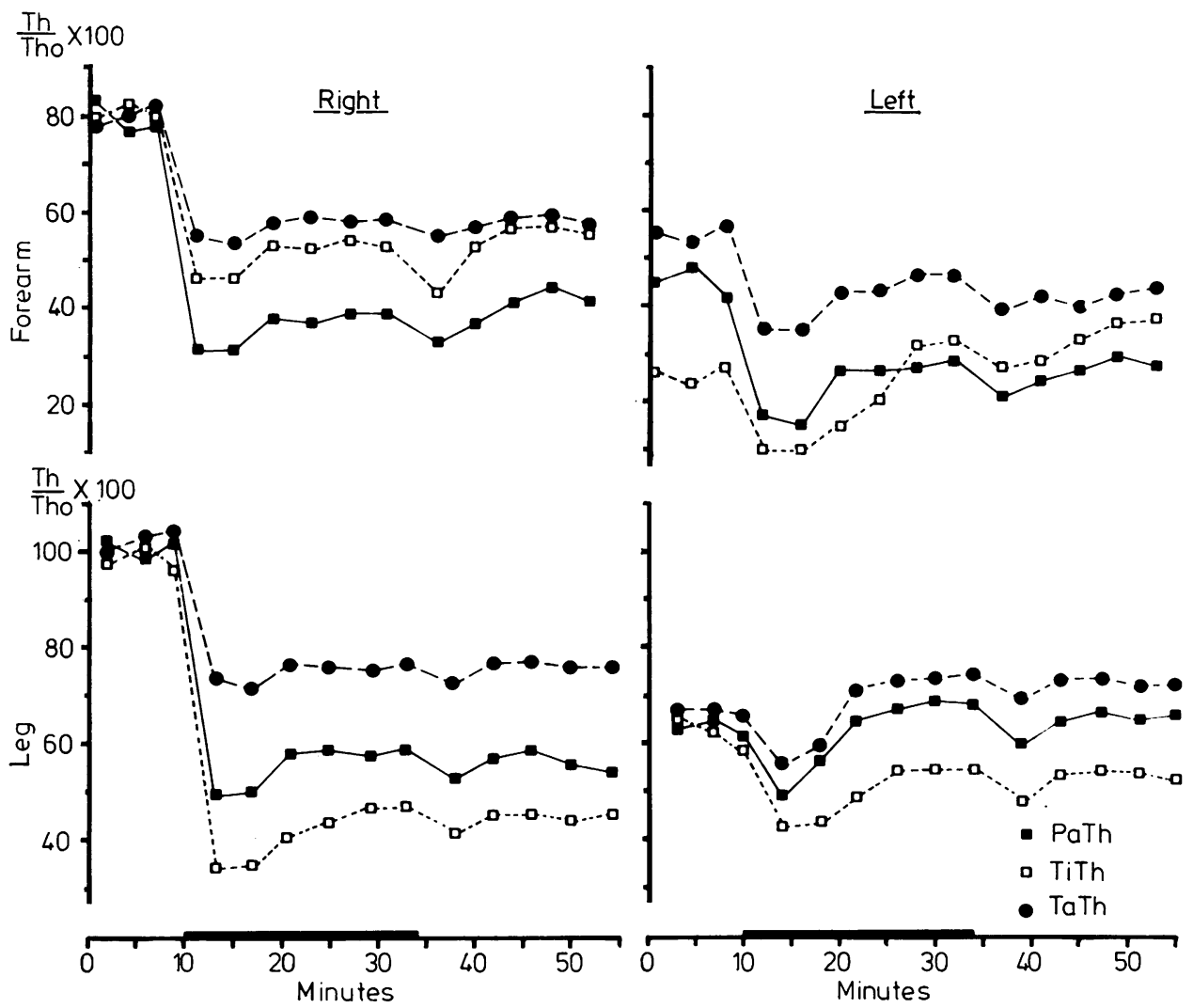

Fig 3 Changes of sensory thresholds outside the distribution area of the stimulated nerve in the four limbs (general thresholds) before, during and after TENS to the right lower limb of a healthy subject with high thresholds before TENS. Symbols as in fig 2.

affected side, were the following: (1) subjects with lower thresholds in the affected side: forearm: tactile threshold: $-35( \pm 4)$; tingling threshold: $-46( \pm 8)$; pain threshold: $-40( \pm 12)$; leg: tactile threshold: $-48( \pm 5)$; tingling threshold: $-40( \pm 8)$; pain threshold: $-45( \pm 10)$; (2) subjects with higher thresholds in the affected side: forearm: tactile threshold: $+25( \pm 5)$; tingling threshold: $+55( \pm 18)$; pain threshold: +35 $( \pm 12)$; leg: tactile threshold: $+35( \pm 7)$; tingling threshold: $+37( \pm 8)$; pain threshold: $+35( \pm 12)$.

In order to maintain the tingling sensation constant, in the subjects with lower thresholds on the affected side, it was necessary to increase the intensity of the current during the first minutes of TENS. The current then had to be decreased because of the onset of unpleasant paraesthesiae or pain. This first period lasted 10-15 minutes ("changing phase" of TENS). During the remain- ing part of stimulation, tingling was steady without relevant changes of the current ("steady phase" of TENS) (table 2).

During the changing phase of TENS in these subjects, the local sensory thresholds increased and then decreased. During the steady phase the thresholds increased again. Twenty minutes after TENS the thresholds were still higher than before TENS (fig 4, table 2). The general sensory thresholds decreased during the changing phase of TENS and increased during the steady phase, remaining close to the level reached for at least 20 min after TENS (fig 5 ; table 2).

In the group of subjects with higher thresholds on the affected side, the intensity of the current had to be decreased after a few minutes of TENS because of the onset of unpleasant paraesthesiae or pain. The current was then increased again as the unpleasant sensations stopped, and the only 
Table 2 Changes of sensory thresholds related to TENS in patients

\begin{tabular}{|c|c|c|c|c|c|c|c|c|c|}
\hline & & & & \multicolumn{3}{|c|}{ Ipsi< Contra (18) } & \multicolumn{3}{|c|}{ Ipsi $>$ Contra (12) } \\
\hline & & & & Ipsi & Contra & $\Delta(I-C)$ & Ipsi & Contra & $\Delta(I-C)$ \\
\hline \multirow[t]{2}{*}{$\begin{array}{l}\text { A: } \\
\text { Changing } \\
\text { phase of } \\
\text { of TENS }\end{array}$} & $\begin{array}{l}\text { General } \\
\text { thresholds }\end{array}$ & $\begin{array}{l}\text { Forearm } \\
\text { Leg }\end{array}$ & $\begin{array}{l}\Delta \mathrm{TaTh} \\
\Delta \mathrm{TiTh} \\
\Delta \mathrm{PaTh} \\
\Delta \mathrm{TaTh} \\
\Delta \mathrm{TiTh} \\
\Delta \mathrm{PaTh}\end{array}$ & $\begin{array}{l}-42 \pm 5 \\
-36 \pm 4 \\
-27 \pm 5 \\
-22 \pm 6 \\
-36 \pm 7 \\
-32 \pm 6\end{array}$ & $\begin{array}{l}-16 \pm 6 \\
-27 \pm 5 \\
-15 \pm 5 \\
-22 \pm 5 \\
-30 \pm 7 \\
-15 \pm 4\end{array}$ & $\begin{array}{l}-56 \pm 8 \\
-55 \pm 8 \\
-52 \pm 7 \\
-48 \pm 8 \\
-50 \pm 8 \\
-62 \pm 7\end{array}$ & $\begin{array}{l}-26 \pm 6 \\
-34 \pm 5 \\
-50 \pm 6 \\
-30 \pm 5 \\
-65 \pm 7 \\
-50 \pm 6\end{array}$ & $\begin{array}{l}-26 \pm 4 \\
-15 \pm 6 \\
-30 \pm 6 \\
-10 \pm 2 \\
-20 \pm 3 \\
-15 \pm 3\end{array}$ & $\begin{array}{r}+19 \pm 7 \\
+36 \pm 8 \\
+15 \pm 5 \\
+15 \pm 5 \\
-8 \pm 7 \\
0 \pm 6\end{array}$ \\
\hline & $\begin{array}{l}\text { Local } \\
\text { thresholds }\end{array}$ & & $\begin{array}{l}\Delta \mathrm{TaTh} \\
\Delta \mathrm{TiTh} \\
\Delta \mathrm{PaTh} \\
\Delta \mathrm{l}\end{array}$ & $\begin{array}{c}+30 \pm 8 \\
+40 \pm 7 \\
+15 \pm 9 \\
+180 \pm 27\end{array}$ & & & $\begin{array}{c}-4 \pm 6 \\
-8 \pm 8 \\
-12 \pm 9 \\
-50 \pm 18\end{array}$ & & \\
\hline \multirow[t]{2}{*}{$\begin{array}{l}\text { B: } \\
\text { Steady } \\
\text { phase of } \\
\text { TENS }\end{array}$} & $\begin{array}{l}\text { General } \\
\text { thresholds }\end{array}$ & $\begin{array}{l}\text { Forearm } \\
\text { Leg }\end{array}$ & $\begin{array}{l}\Delta \mathrm{TaTh} \\
\Delta \mathrm{TiTh} \\
\Delta \mathrm{PaTh} \\
\Delta \mathrm{TaTh} \\
\Delta \mathrm{TiTh} \\
\Delta \mathrm{PaTh}\end{array}$ & $\begin{array}{l}+12 \pm 4 \\
+25 \pm 5 \\
+35 \pm 4 \\
+12 \pm 3 \\
+20 \pm 3 \\
+38 \pm 3\end{array}$ & $\begin{array}{r}+20 \pm 3 \\
+12 \pm 4 \\
+25 \pm 5 \\
+5 \pm 4 \\
+5 \pm 4 \\
+18 \pm 3\end{array}$ & $\begin{array}{l}-43 \pm 3 \\
-33 \pm 2 \\
-30 \pm 3 \\
-41 \pm 4 \\
-25 \pm 4 \\
-35 \pm 4\end{array}$ & $\begin{array}{l}-22 \pm 4 \\
-27 \pm 4 \\
-42 \pm 3 \\
-24 \pm 4 \\
-54 \pm 4 \\
-41 \pm 3\end{array}$ & $\begin{array}{r}-10 \pm 4 \\
+5 \pm 3 \\
-15 \pm 2 \\
+5 \pm 3 \\
-8 \pm 3 \\
+3 \pm 4\end{array}$ & $\begin{array}{r}+13 \pm 5 \\
+23 \pm 3 \\
+8 \pm 4 \\
+6 \pm 3 \\
-9 \pm 4 \\
-9 \pm 3\end{array}$ \\
\hline & $\begin{array}{l}\text { Local } \\
\text { thresholds }\end{array}$ & & $\begin{array}{l}\Delta \mathrm{TaTh} \\
\Delta \mathrm{TiTh} \\
\Delta \mathrm{PaTh} \\
\Delta \mathrm{I}\end{array}$ & $\begin{array}{l}+60 \pm 8 \\
+65 \pm 5 \\
+31 \pm 8 \\
+62 \pm 8\end{array}$ & & & $\begin{array}{l}+25 \pm 7 \\
+31 \pm 7 \\
+17 \pm 8 \\
+10 \pm 3\end{array}$ & & \\
\hline \multirow[t]{2}{*}{$\begin{array}{l}\text { C: } \\
\text { After } \\
\text { TENS }\end{array}$} & $\begin{array}{l}\text { General } \\
\text { thresholds }\end{array}$ & Forearm & $\begin{array}{l}\Delta \mathrm{TaTh} \\
\Delta \mathrm{TiTh} \\
\Delta \mathrm{PaTh} \\
\Delta \mathrm{TaTh} \\
\Delta \mathrm{TiTh} \\
\Delta \mathrm{PaTh}\end{array}$ & $\begin{array}{l}+12 \pm 6 \\
+25 \pm 5 \\
+35 \pm 6 \\
+10 \pm 5 \\
+25 \pm 4 \\
+35 \pm 5\end{array}$ & $\begin{array}{r}-5 \pm 3 \\
-5 \pm 4 \\
+20 \pm 3 \\
-12 \pm 4 \\
-18 \pm 4 \\
-1 \pm 3\end{array}$ & $\begin{array}{r}-17 \pm 5 \\
-16 \pm 5 \\
-25 \pm 5 \\
-26 \pm 6 \\
+8 \pm 4 \\
-9 \pm 3\end{array}$ & $\begin{array}{l}-21 \pm 5 \\
-25 \pm 4 \\
-40 \pm 5 \\
-25 \pm 6 \\
-55 \pm 4 \\
-45 \pm 5\end{array}$ & $\begin{array}{r}-12 \pm 5 \\
+8 \pm 5 \\
-12 \pm 6 \\
+5 \pm 4 \\
-7 \pm 3 \\
+1 \pm 3\end{array}$ & $\begin{array}{r}+15 \pm 4 \\
+22 \pm 4 \\
+7 \pm 5 \\
+5 \pm 4 \\
-11 \pm 3 \\
-11 \pm 2\end{array}$ \\
\hline & $\begin{array}{l}\text { Local } \\
\text { thresholds }\end{array}$ & & $\begin{array}{l}\Delta \mathrm{TaTh} \\
\Delta \mathrm{TiTh} \\
\Delta \mathrm{PaTh}\end{array}$ & $\begin{array}{l}+40 \pm 12 \\
+50 \pm 10 \\
+25 \pm 9\end{array}$ & & & $\begin{aligned}+14 & \pm 11 \\
+12 & \pm 8 \\
+3 & \pm 7\end{aligned}$ & & \\
\hline
\end{tabular}

Mean \pm SE $\quad \Delta$ Ths $=\left(\right.$ Ths $\left.-\mathrm{Ths}_{0} / \mathrm{Ths}_{0}\right) \times 100 ; \Delta(\mathrm{I}-\mathrm{C})=[\operatorname{Tns}(\mathrm{Ipsi})-\mathrm{Ths}($ Contra $\left.) / \mathrm{Ths}(\mathrm{Ips})] \times 100 ;\right) \quad \Delta \mathrm{I}=\left(\mathrm{I}-\mathrm{I}_{0} / \mathrm{I}_{0}\right) \times 100$.

4: General and local sensory thresnolds and current intensity of TENS during the changing phase. The sample shown was the change at the 4th min of TENS.

B: General and local sensory thresholds and current intensity of TENS during the steady pnase. Figure given is the average of the values during the last $10 \mathrm{~min}$ of TENS.

C: General and local sensory thresholds after TENS. Figure given is the average of the values during the first $10 \mathrm{~min}$ after TENS.

Ipsi<Contra: subjects with sensory thresholds on the pain side (ipsi) lower than on the opposite side (Contra). Ipsi $>$ Contra: subjects with higher sensory tl resholds on the pain side than on the opposite side. In brackets: number of subjects. TENS was always applied to the painful leg. Thresholds are expressed as changes $(\Delta T h s)$ from the values before TENS $\left(T_{h} s_{0}\right) . \Delta I=$ changes of the intensity of TENS from $I_{0} . I_{0}$ is the value of current at which the subjects begin feeling a sharp tingling sensation. $\Delta(\mathrm{I}-\mathrm{C})$ : difference between the sensory thresholds on the pain side and the sensory thresnolds on the opposite side. Other abbreviations as in table 1.

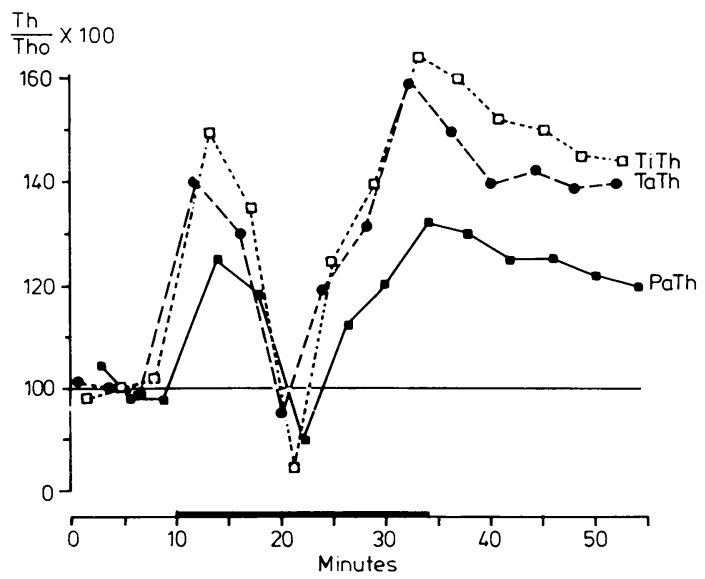

Fig 4 Changes of sensory thresholds in the distributicn area of the $n$ cutaneus surae medialis (local thresholds) in a patient with lower sensory thresholds in the side ipsilateral to the affected limb. The affected limb was the right lower limb, where TENS was applied. Symbols as in fig 1. 

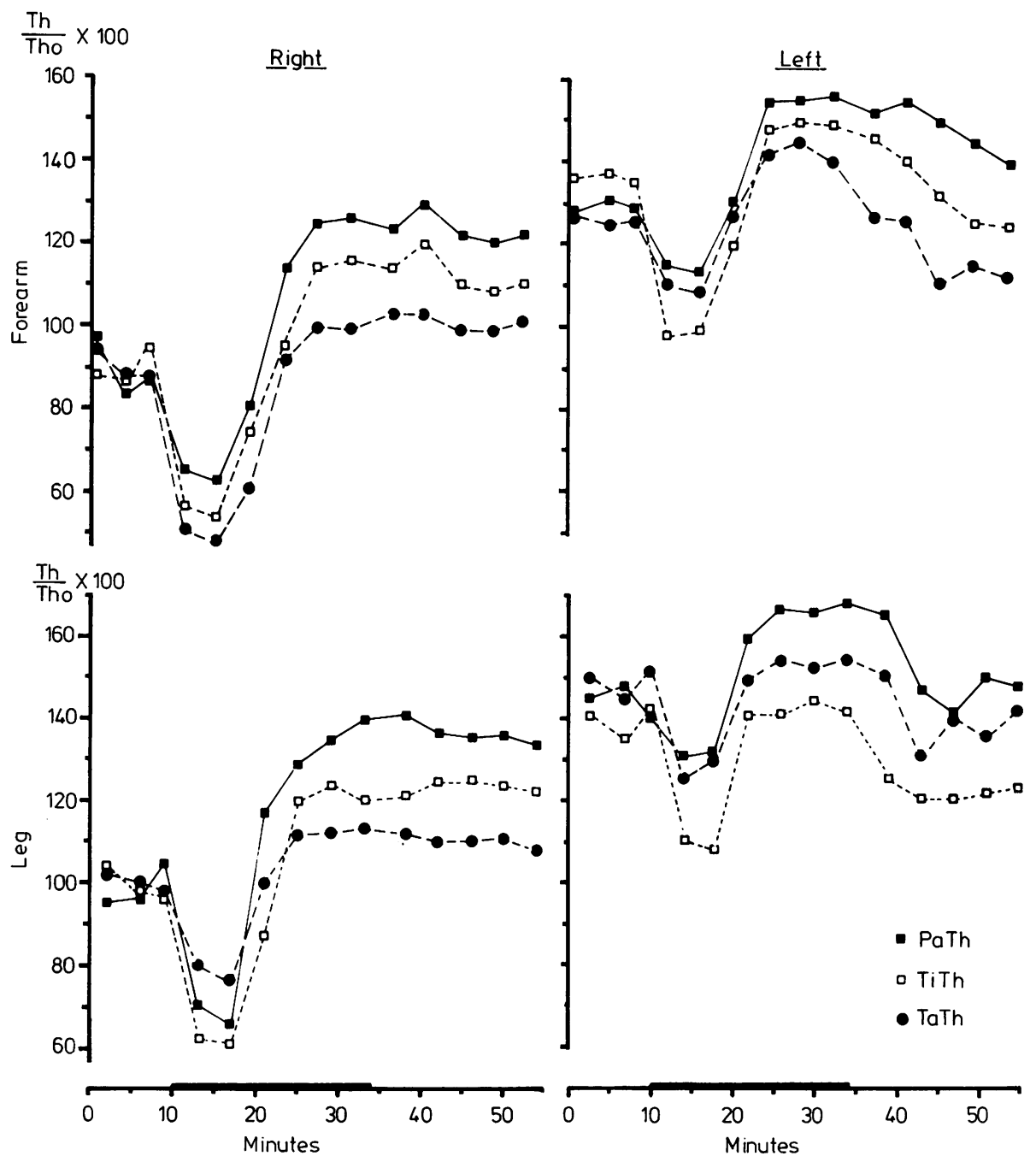

Fig 5 Changes of sensory thresholds in the four limbs (general thresholds) in the same subject as fig 4. Symbols as in fig 2.

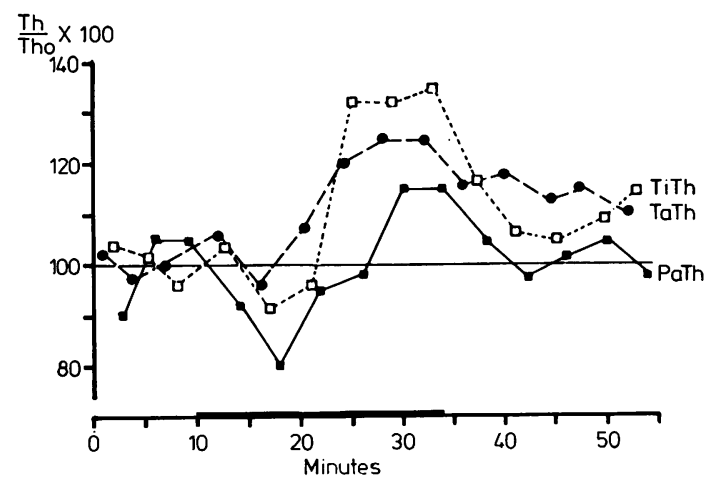

Fig 6 Changes of sensory thresholds in the distribution area of the $n$ cutaneus surae medialis (local thresholds) in a patient with higher sensory thresholds in the side ipsilateral to the affected limb. The affected limb was the right lower limb, where TENS was applied. Symbols as in fig 1. 

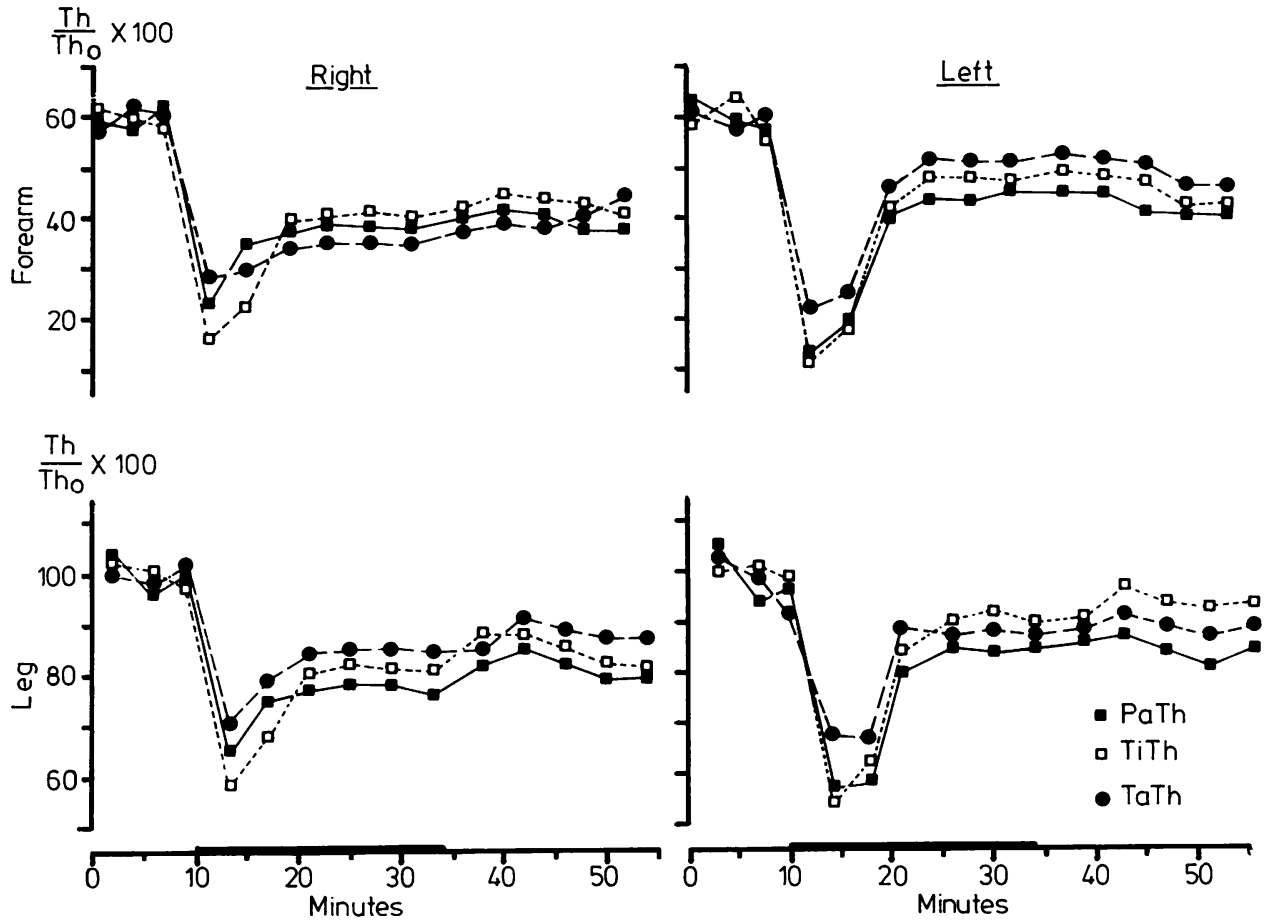

Fig 7 Changes of sensory thresholds in the four limbs (general thresholds) in the same subject as fig 6. Symbols as in fig 2.

remaining sensation was an almost imperceptible tingling (changing phase of TENS: first 10-14 min). The intensity of the current during the last minutes of TENS (steady phase of TENS) was close to the value at which subjects began feeling a sharp tingling (table 2).

Local sensory thresholds showed no significant changes during the changing phase. During the steady phase the increase was lower than in the group with lower thresholds in the affected side. The values of the thresholds returned close to the values observed before TENS within 10 minutes after TENS (fig 6, table 2). General sensory thresholds showed a sharp decrease on the affected side and remained unchanged until the end of the experiment (fig 7 , table 2 ).

\section{Discussion}

We have distinguished two phases during TENS: the changing phase, during which the intensity of TENS must be changed in order to cause a constant degree of tingling; and the steady phase, during which a constant intensity of stimulation causes a constant degree of tingling. As expected,
TENS induces changes in sensory thresholds within the territory of the stimulated nerve, and it also induces changes in thresholds throughout the body.

In TENS, the interval between two pulses is $20 \mathrm{~ms}$, which is over 20 times the absolute refractory period of large myelinated fibres. The amplitude of the compound action potential begins decreasing only when the interval between two stimuli is less than three times the refractory period; the conduction-time decreases when the interval is less than four times the refractory period. Thus, in our experimental conditions complete recovery of function of large diameter fibres occurs. ${ }^{18}$ For this reason the changes of the sensations induced by TENS are not due to a direct effect on nerve fibres.

A relationship was observed between local and general effects on sensory thresholds: the increase in local thresholds was higher when a concomitant increase in general thresholds was present, but the increase in local thresholds was lower when the general thresholds decreased. TENS induces an increase of local thresholds probably acting both at the periphery and centrally. 
The peripheral mechanisms in large diameter fibres $(\mathbf{A} \beta)$ may be a collision between the antidromic pulses generated by TENS and the orthodromic pulses that occur during the determination of tactile and tingling thresholds. During TENS 50 action potentials per second flow in large fibres with an interval between pulses of $20 \mathrm{~ms}$; the determination of thresholds was carried out with pulses at a frequency of $250 \mathrm{~Hz}$ and therefore the interval between two pulses is $4 \mathrm{~ms}$. Considering the action potential and the absolute refractory period of $\mathrm{A} \beta$ fibres, ${ }^{19} 20$ we can deduce that $0-2$ action potentials out of six collide and extinguish. Peripheral mechanisms of inhibition on small diameter fibres, if present, are of little importance, because TENS with the parameters which we used mainly stimulates large fibres.

As regards the general thresholds outside the distribution area of the stimulated nerve, the effects of TENS may be excitatory or inhibitory. During the changing phase a general excitatory condition which causes lowering of the thresholds is prevalent in all subjects. This condition is probably due to a central excitatory state. During the steady phase the threshold trend is related to the values observed before TENS. In normal subjects with high thresholds, a slight excitatory state remains, so that the thresholds do not reach the values observed before TENS; in subjects with low thresholds, an inhibitory state is prevalent, so that the thresholds become higher than before TENS.

In the patients we noted a difference in the sensory thresholds of the two sides of the body before TENS. The side with chronic pain showed higher or lower thresholds than the opposite side. In these patients, as in normal subjects, the general thresholds showed different patterns according to the values observed before TENS. When the thresholds were higher on the painful side, the thresholds decreased during the changing phase, and remained at a low level. However, when the thresholds before TENS were lower on the painful side, after a decrease in the changing phase, the thresholds strongly increased during the steady phase. After TENS, the threshold difference between the two sides of the body ("lateralisation") was reduced. As a consequence, patients' thresholds became more similar to those of the normal subjects than before TENS. Pain relief induced by TENS may be related to this fact.

The authors thank Dr PW Nathan for his helpful criticism.

\section{References}

1 Wall PD, Sweet WH. Temporary abolition of pain in man. Science 1967; 155:108-9.

2 Long DM, Hagfors N. Electrical stimulation in the nervous system: the current status of electrical stimulation of the nervous system for relief of pain. Pain 1975; 1:109-23.

3 Andersson SA. Pain control by sensory stimulation. In: Bonica JJ, Liebeskind JC, Albe-Fessard $\mathrm{D}$ eds. Advances in Pain Research and Therapy, Vol 3. New York: Raven Press 1979; 569-85.

4 Sjölund BH, Eriksson MBE. Stimulation techniques in the management of pain. In: Kosterlitz HW and Terenius LY, eds. Pain and Society (Dahlem Workshop Report). Weinheim: Verlag Chemie 1980; 415-30.

5 Bates JAV, Nathan PW. Transcutaneous electrical nerve stimulation for chronic pain. Anaesthesia 1980; 35:817-22.

6 Procacci P, Zoppi M, Maresca M, Francini F. Hypoalgesia induced by transcutaneous electrical stimulation. A physiological and clinical investigation. J Neurosurg Sci 1977; 21:221-8.

7 Taub A, Campbell JN. Percutaneous local electrical analgesia; peripheral mechanisms. In: Bonica JJ, ed. Advances in Neurology, Vol 4. New York: Raven Press 1974; 727-32.

8 Chapman CR, Wilson ME, Gehrig JD. Comparative effects of acupuncture and transcutaneous stimulation on the perception of painful dental stimuli. Pain 1976; 2:265-83.

9 Clark WC, Hall W, Yang J. Changes in thermal discriminability and pain report criterion after acupunctural or transcutaneous electrical stimulation. In: Bonica JJ and Albe-Fessard D, eds. Advances in Pain Research and Therapy, Vol 1. New York: Raven Press 1976: 769-73.

10 Callaghan M, Sternbach RA, Nyquist JK, Timmermans $\mathrm{G}$. Changes in somatic sensitivity during transcutaneous electrical analgesia. Pain 1978; 5:115-27.

11 Della Corte M, Procacci P, Bozza G, Buzzelli G. A study on the cutaneous pricking pain threshold in normal man. Arch Fisiol 1965; 64:141-70.

12 Procacci P, Francini F, Zoppi M, Maresca M. Cutaneous pain threshold changes after sympathetic block in reflex dystrophies. Pain 1975; 1: 167-75.

13 Francini F, Zoppi M, Maresca M, Procacci P. Skin potential and EMG changes induced by cutaneous electrical stimulation. I. Normal man in arousing and non-arousing environment. Appl Neurophysiol 1979; 42:113-24.

14 Procacci P, Francini F, Maresca M, Zoppi M. Skin potential and EMG changes induced by cutaneous electrical stimulation. II. Subjects with reflex sympathetic dystrophies. A ppl Neurophysiol 1979; 42:125-34.

15 Procacci P, Della Corte M, Zoppi M, Romano S, 
Maresca M, Voegelin MR. Pain threshold measurements in man. In: Bonica JJ, Procacci P, Pagni CA, eds. Recent Advances on Pain. Springfield: Charles C Thomas 1974; 105-47.

16 McCarty DJ. Arthritis and Allied Conditions. Philadelphia: Lea and Febiger 1979.

17 Procacci P, Zoppi M, Dorigo B, et al. La soglia del dolore cutaneo negli arti inferiori determinata con 1'algometria termica. Minerva Cardioangiol $1971 ; 19: 593-8$.
18 Buchtal F, Rosenfalck A. Evoked action potentials and conduction velocity in human sensory nerves. Brain Res 1966; 3:1-122.

19 Paintal AS. Participation by pressure-pain receptors of mammalian muscles in the flexion reflex. J Physiol (Lond) 1961; 156:498-514.

20 Kalia M, Senapati JM, Parida B, Panda A. Reflex increase in ventilation by muscle receptors with non-medullated fibers (C fibers). J Appl Physiol 1972; 32:189-93. 